\title{
Congestion phenomena caused by matching pennies in evolutionary games
}

\author{
György Szabó ${ }^{1,2}$ and Attila Szolnoki ${ }^{1}$ \\ ${ }^{1}$ Institute of Technical Physics and Materials Science, Research Centre for Natural Sciences, Hungarian Academy of Sciences, \\ P.O. Box 49, H-1525 Budapest, Hungary \\ ${ }^{2}$ Regional Knowledge Centre, Eötvös University, Irányi Dániel utca 4, H-8000 Székesfehérvár, Hungary
}

(Received 18 August 2014; published 5 March 2015)

\begin{abstract}
Evolutionary social dilemma games are extended by an additional matching-pennies game that modifies the collected payoffs. In a spatial version players are distributed on a square lattice and interact with their neighbors. First, we show that the matching-pennies game can be considered as the microscopic force of the Red Queen effect that breaks the detailed balance and induces eddies in the microscopic probability currents if the strategy update is analogous to the Glauber dynamics for the kinetic Ising models. The resulting loops in probability current breaks symmetry between the chessboardlike arrangements of strategies via a bottleneck effect occurring along the four-edge loops in the microscopic states. The impact of this congestion is analogous to the application of a staggered magnetic field in the Ising model; that is, the order-disorder critical transition is wiped out by noise. It is illustrated that the congestion induced symmetry breaking can be beneficial for the whole community within a certain region of parameters.
\end{abstract}

DOI: 10.1103/PhysRevE.91.032110

PACS number(s): 02.50.Le, 05.70.Ln, 87.23.Kg, 89.65.-s

\section{INTRODUCTION}

Multiagent evolutionary games on networks [1-4] constitute a frequently used approach to study evolutionary processes characteristic to biological and social systems. In these models players are located on the sites of a network (or lattice) and their strategies represent biological entities or individual choices. For most of the cases the pair interactions among players are characterized by two-player games [5].

In these evolutionary games a uniform, symmetric, twoplayer, two-strategy game defines the payoffs for both players [6]. In that simple case we can distinguish only four possible strategy pairs (henceforth strategy profiles) for the elementary interactions. In Fig. 1 these strategy profiles are illustrated by large boxes with white and black bullets representing the two possible strategies for each player. The actual payoffs are denoted within these bullets. For the prisoner's dilemma, as for the most challenging social dilemmas, the black and white bullets refer to defection $(D)$ and cooperation (C) strategies, respectively, while for the matching-pennies game these symbols denote head and tail (two sides of a coin) states. Henceforth, we use the abbreviated nomination of $C$ and $D$ strategies and the traditional notation of payoffs introduced for the investigation of social dilemmas $[7,8]$.

The mentioned games represent two significantly different behaviors, as illustrated in the flow diagrams where the edges connect those strategy profiles that can be transformed into each other by a single strategy change of a player. For the symmetric two-player, two-strategy games the payoff variations of the active player from both the $D D$ and $C C$ strategy profiles are equivalent. In fact, this is the reason why the sum of the payoff variations of active players along the single loop is zero independently of the values of $S$ and $T$. Consequently, all the symmetric two-player two-strategy games are potential games [9-11]. For the potential games one can find a potential (as a function of strategy profile) summarizing the driving force for the unilateral strategy changes on the analogy of the potential energy in physical systems. For the multistrategy systems potential can exist if the above condition is satisfied along all the possible loops in the space of strategy profiles. The existence of potential is accompanied with some general features. Namely, the maximal value of potential is achieved at a pure Nash equilibrium; additional pure Nash equilibria are represented by nodes without outgoing edges; and the absence of directed loops is forbidden in the flow diagram. For the potential games the latter feature allows that one can arrive to one of the pure Nash equilibria when following the direction of arrows from any initial state for the multistrategy and/or multiagent games $[12,13]$.

If a multiagent system is built up from two-player potential games, then the potential for the whole system will be the sum of the pair potentials. Blume $[14,15]$ has shown that the evolutionary potential games evolve into a Gibbs ensemble if the evolution is controlled by a logit rule [16] that is similar to the Glauber [17] or Metropolis [18] dynamics introduced for the investigation of kinetic Ising models. In other words, all these multiagent systems with symmetric two-strategy interactions are equivalent to an Ising model [6,19-21].

On the contrary, the flow diagram of matching-pennies game represents a fundamentally different interaction. In that case the players are not equivalent and one of the players is always motivated to change her strategy. As a result, there is a uniform payoff increase (driving force) along the four edges that represents a directed loop in the flow diagram. For this game, potential does not exist and the single Nash equilibrium is a mixed strategy profile where both players choose their strategy at random $[22,23]$. The matching-pennies game can be interpreted as a driving force creating circular transitions in the flow diagram and probability current loops in the dynamical graphs that can be quantified by evaluating the entropy production [21,24]. The relevance of a conceptually similar circular transition in biological systems was first described by van Valen $[25,26]$, who named this effect the Red Queen mechanism. Similar endless races can be observed in social systems between buyers and sellers [27], property owners and criminals [28], males and females [29,30], or conformists and rebels [31]. The consideration of an evolutionary multiagent system on network with this type of interaction prescribes 

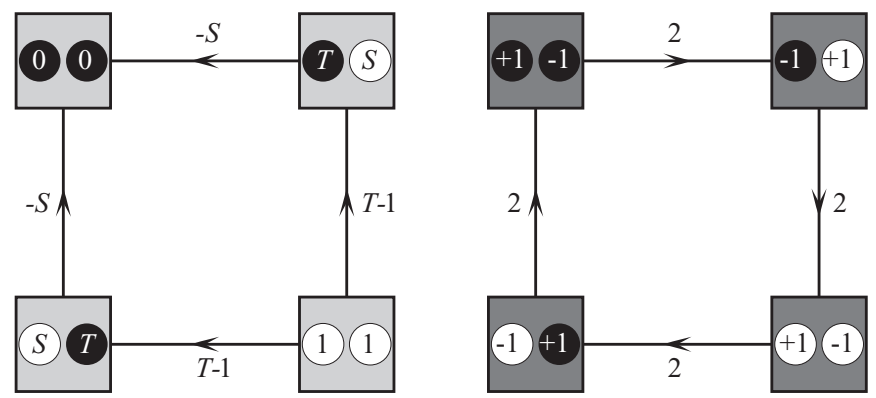

FIG. 1. Flow diagrams for two-payer, two-strategy games. The left and right diagrams show the payoffs for the prisoner's dilemmas ( $S<0$ and $T>1$ ) and matching-pennies game. Arrows along the edges point towards the preferred strategy profile from the viewpoint of a player who modifies unilaterally her strategy.

two kinds of players distributed on bipartite graphs or lattices [32].

The evolutionary matching-pennies game and its combination with an anticoordination game were considered previously for several structured populations [21]. In the present paper we extend these analyses to systems where the mentioned probability current loops are distorted by bottleneck effects which can modify the macroscopic behavior significantly.

\section{THE MODEL}

Now we study spatial evolutionary games with players located on a square lattice with periodic boundary conditions. Each player has two strategies and collects income by playing games with the four nearest neighbors. The pair interaction is composed of a symmetric two-player, two-strategy game (a social dilemma with $T-S$ parametrization) and a matchingpennies game. Our analysis focuses on the region of hawk-dove games $(T>1$ and $S>0)$ where chessboardlike strategy arrangements occur for myopic or Glauber type strategy updates preferred in statistical physics [21,33,34].

Both the suitable description of the chessboardlike strategy arrangements and the application of the matching-pennies game require the division of the square lattice into two equivalent sublattices denoted as $\alpha=X$ or $Y$, resembling the black and white squares on a chessboard. For this sublattice division each site $x(x \in X)$ is surrounded by four nearest neighbors belonging to the opposite sublattice $(x+\delta \in Y)$ and vice versa. It is convenient to denote the strategies of players at sites $x$ and $y$ by unit vectors as

$$
\mathbf{s}_{x}, \mathbf{s}_{y}=D=\left(\begin{array}{l}
1 \\
0
\end{array}\right), \quad \text { or } \quad C=\left(\begin{array}{l}
0 \\
1
\end{array}\right) .
$$

Using this traditional notation, the player's income can be given by a sum of matrix products

$$
u_{x}=\sum_{\delta} \mathbf{s}_{x} \cdot \mathbf{A} \mathbf{s}_{x+\delta}, \quad u_{y}=\sum_{\delta} \mathbf{s}_{y} \cdot \mathbf{B} \mathbf{s}_{y+\delta}
$$

where the summation runs over the four nearest neighbor sites belonging to the opposite sublattice. According to the model definition the pair interactions of nearest neighbors are the composition of a social dilemma and a cyclic game. The corresponding payoff matrix is given as

$$
\mathbf{A}=\left(\begin{array}{cc}
\varepsilon & T-\varepsilon \\
S-\varepsilon & 1+\varepsilon
\end{array}\right), \quad \mathbf{B}=\left(\begin{array}{cc}
-\varepsilon & T+\varepsilon \\
S+\varepsilon & 1-\varepsilon
\end{array}\right)
$$

where $\varepsilon$ quantifies the strength of matching-pennies component.

During the elementary step of the evolutionary process a player is chosen randomly and she modifies her strategy from $\mathbf{s}_{x}$ to $\mathbf{s}_{x}^{\prime}$ with a probability depending on the payoff difference $\left(u_{x}^{\prime}-u_{x}\right)$ between the final and the initial states. Namely, the strategy update probability is given as

$$
W\left(\mathbf{s}_{x} \rightarrow \mathbf{s}_{x}^{\prime}, \mathbf{s}_{-x}\right)=\frac{1}{1+\exp \left[\left(u_{x}-u_{x}^{\prime}\right) / K\right]},
$$

where the strategy profile for the rest of players, denoted traditionally as $\mathbf{s}_{-x}$, remains unchanged.

This transition favors the state providing a higher payoff for the player $x$, and a similar rule is applied for the players of the opposite sublattice. Notice that $W\left(\mathbf{s}_{x} \rightarrow \mathbf{s}_{x}^{\prime}, \mathbf{s}_{-x}\right) \simeq 1$ (or 0 ) if $u_{x} \ll u_{x}^{\prime}$ (or $u_{x} \gg u_{x}^{\prime}$ ), while the "width" of the transient region is proportional to $K$ that measures the magnitude of noise or the strength of selection. It is noteworthy that the probability of strategy change [given by Eq. (4)] is equivalent to those suggested by Glauber [17] for the kinetic Ising model.

For $\varepsilon=0$ the above system satisfies the condition of potential games [9] because the sum of payoff variation along the single loop is zero, as illustrated in Fig. 1. In fact, the kinetic Ising model and the corresponding two-strategy evolutionary potential games are equivalent; thus, the coupling constant $(J)$ between the neighboring spins $\left(s_{x}= \pm 1\right)$ and the strength $(h)$ of the external magnetic field can be expressed by the payoff parameters as $J=(1-S-T) / 4$ and $h=1+S-T$, while $K$ corresponds to the temperature in the thermodynamical system $[6,11,19,20]$. The effect of $\varepsilon$ on the transition from the antiferromagnetic to the paramagnetic phase as a function of $K$ is investigated in a recent paper for the absence of external field $h$ [21]. It is found that the disturbance of the matching-pennies component reduces the critical value of $K$, while the universal (Ising type) features of the critical transition are preserved.

In the present work we explore how the stationary states of an evolutionary social dilemmas are influenced by the matching-pennies game in the presence of an external field $(h \neq 0)$ representing games with $S \neq T-1$. In the literature of Ising models [35], it is well described that at zero temperature the ordered antiferromagnetic state is transformed into the preferred ferromagnetic state if $h$ exceeds a threshold value proportional to $J$ (the prefactor depends on the number of neighbors determined by the lattice structure). On the other hand, by increasing temperature $K$ the system exhibits an Ising type critical phase transition from the ordered antiferromagnetic state to the disordered spin arrangement at a critical point $\left(K_{c}\right)$ decreasing with $|h|$ [36-38]. Now it will be shown that the latter robust critical transition is smoothed out for $|\varepsilon|>0$. For this purpose we first study a two-player game exhibiting the congestion phenomenon that results in macroscopic changes in the multiplayer systems. 


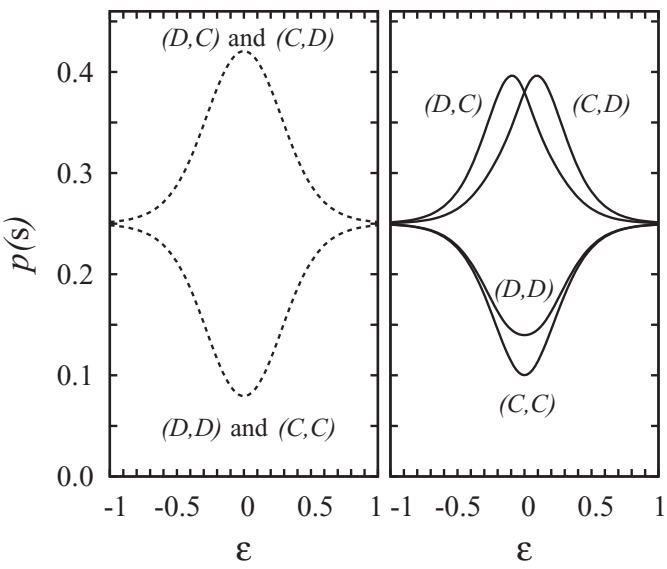

FIG. 2. Probabilities of strategy pairs as a function of $\varepsilon$ for $T=$ $1.5, S=0.5$ (left panel, dashed lines) and $T=1.4, S=0.3$ (right panel, solid lines) at $K=0.3$.

\section{CONGESTION FOR TWO-PLAYER SYSTEM}

In this section we study a two-player game with the same dynamical rule introduced in the previous section. In that case the system has only four microscopic states $[\mathbf{s}=(D, D),(D, C),(C, D)$, or $(C, C)]$ and the corresponding probabilities $p(\mathbf{s})$ in the stationary state can be determined numerically by solving the suitable equations of motion for all values of payoff parameters and noise levels. In the absence of neighborhood, we can apply a simplified version of the traditional pair approximation (for a brief survey, see $[6,39])$. Figure 2 compares the configuration probabilities in dependence of $\varepsilon$ for the all the four possible strategy pairs at a fixed noise level. The dashed lines in the left panel of Fig. 2 represent the behavior when the $(D, D)$ and $(C, C)$ strategy pairs appear with the same probability for arbitrary values of $\varepsilon$. This is equivalent to the $h=0$ case for the terminology of the Ising model. Here $p(D, C)=p(C, D)$ for any values of $\varepsilon$ and all four configuration probabilities tend to $1 / 4$ if the matching-pennies components dominate the game, as discussed in Ref. [21]. The latter behavior reflects the fact that the matching-pennies game has only one mixed Nash equilibrium when both players choose their strategies at random. Notice furthermore that the variations in the configuration probabilities are even functions of $\varepsilon$.

Significantly different behavior can be observed if $1+$ $S-T=h \neq 0$ even for $\varepsilon=0$. The right plot of Fig. 2 shows a situation when the preferred $(C, D)$ and $(D, C)$ strategies are present with the same high probability, while $p(D, D)>p(C, C)$ is obtained for the payoff parameters given in the caption of Fig. 2. It is emphasized that in the absence of a matching-pennies component the system satisfies the condition of detailed balance; that is, the forward and backward transitions appear with the same probabilities along the four edges of the flow diagram, as illustrated in the left plot of Fig. 3.

The presence of the matching-pennies component, however, breaks the detailed balance and induces a probability current through the four-edge loop with a strength characterized by the difference of transition frequencies between the forward and backward directions along the four edges. In the
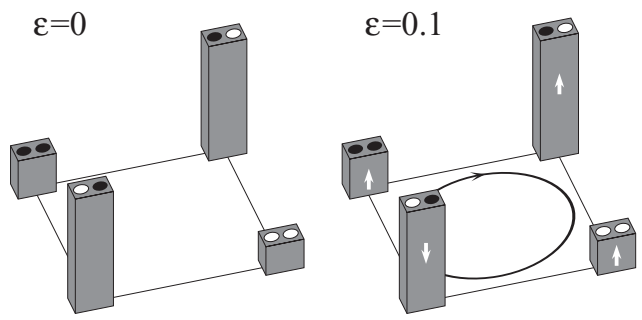

FIG. 3. Configuration probabilities are illustrated by the heights of columns for the absence (left plot) and presence (right plot) of a matching-pennies component for $T=1.4$ and $S=0.3$. In the right plot the directed ellipse illustrates the emergence of a probability current loop that modifies the configuration probabilities via a bottleneck effect and the white arrows on the faces of the columns show how the probabilities change due to the congestion phenomenon.

steady state these probability currents should be equivalent due to Kirchhoff laws [40]. This uniform probability current modifies the configuration probabilities $p(\mathbf{s})$ and destroys the equivalence between $(D, C)$ and $(C, D)$ configurations because the $(D, D)$ and $(C, C)$ states represent different barriers (widths of bottleneck) for the probability current flow. As a result, $p(D, C)[p(C, D)]$ increases [decreases] linearly with with the strength of the matching-pennies component for small values of $\varepsilon$. The white arrows in Fig. 3 show that at the bottleneck states the corresponding probabilities increase because it happens before the narrower bottleneck, while the probability of the fourth should be reduced because $\sum_{\mathbf{s}} p(\mathbf{s})=1$.

Notice that the linear dependence on $\varepsilon$ implies that the behaviors of $p(D, C)$ and $p(C, D)$ are exchanged when reversing the sign of $\varepsilon$, which is accompanied with a reversal of probability current, too. For high values of $\varepsilon$ the system tends towards a uniform strategy distribution, as mentioned above. Consequently, if $\varepsilon$ is increased, then $p(C, D)$ exhibits a local maximum at $\varepsilon \simeq 0.1$ if $T=1.4, S=0.3$, and $K=0.3$. For the opposite sign of $\varepsilon$ the variations of $p(D, C)$ and $p(C, D)$ are exchanged, as shown in Fig. 2. The results of this congestion phenomenon resembles the impact of a staggered magnetic field $h_{s}\left(h_{s}\right.$ is positive for the sites of sublattice $X$ and negative on the sublattice $Y$ ) for the antiferromagnetic Ising models that prefers one of the ordered arrangements to the other.

The driving effect of the matching-pennies component seems to be similar to the effect of external electric field in driven lattice gas models [41]. For these models the external field drives the system out off the Boltzmann distribution [42] and generates a permanent particle transport when periodic boundary conditions are applied. It breaks inherent symmetries [43] and enhances the effect of noise and the impact of local inhomogeneities [44]. In the case of repulsive interaction, where a similar chessboardlike ordered phase is formed in the half-filled lattice $[45,46]$, the order-disorder phase transition is modified due to the particle transport [47]. Last, we note that the effect of bottlenecks along a ring was recently studied by considering the asymmetric exclusion process [48], which is the simplest version of the driven lattice gases. The latter investigation is motivated by biological experiments indicating unidirectional circular ribosome translocations along messenger RNA loops with defects or slow codons in cells [49]. 


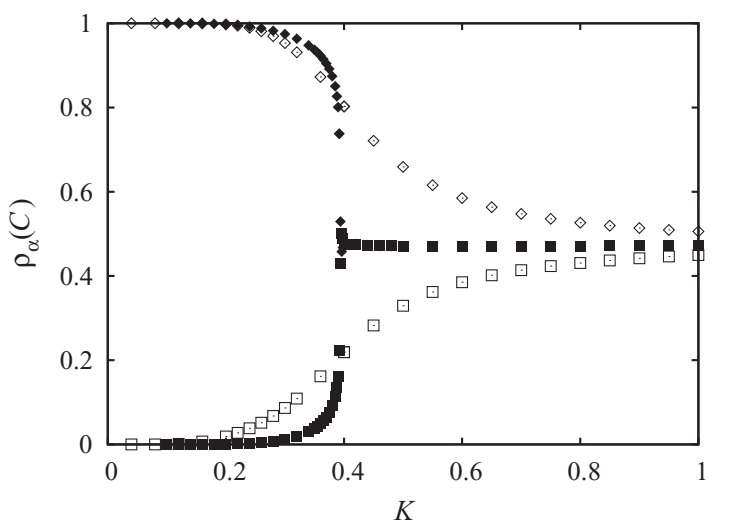

FIG. 4. Frequency of strategy $C$ as a function of $K$ in sublattice $X$ (open diamonds) and $Y$ (open boxes) for $T=1.4, S=0.3$, and $\varepsilon=0.1$. The solid symbols indicate the same quantities for $\varepsilon=0$.

The application of the matching-pennies component in evolutionary games induces local probability currents within many significantly shorter loops that can be quantified even for two neighboring players when considering the transitions among the four possible strategy profiles for most of their quenched neighborhood [21].

\section{CONGESTION FOR A MULTIPLAYER SYSTEM}

The above-mentioned weak effect of the matching-pennies component remains valid for each pair and it is amplified for the multiagent games. As a result, the macroscopic system will evolve into the preferred ordered strategy distribution at sufficiently low noise levels. This fact is illustrated in Fig. 4, where the frequency of strategy $C\left[\rho_{\alpha}(C)\right]$ is plotted as a function of noise level $K$ for both sublattices $(\alpha=$ $X, Y)$. For $\varepsilon=0$ this system shows an Ising type critical transition at $K_{c}=0.3922(1)$ from the sublattice ordered strategy arrangement to a disordered state [21]. This critical transition, however, is smoothed out if the game includes the matching-pennies component.

Open symbols in Fig. 4 show the Monte Carlo (MC) results we obtained for $\varepsilon=0.1$ on a square lattice with a linear size $L=400$. Each full MC step (MCS) gives a chance for every player to change its strategy once on average. To get reliable simulation results the sampling and relaxation times are chosen to be $t_{s}=t_{r}=10000 \mathrm{MCS}$. When reversing the sign of $\varepsilon$, the strategy occupations in the sublattices are exchanged. As we argued, this phenomenon is a straightforward consequence of the congestion effect we described in the two-player model. Furthermore, the sublattice occupations tend toward the prediction of the Ising model if $\varepsilon \rightarrow 0$ in a way resembling the vanishing of the staggered magnetic field. Evidently, we have to use significantly larger system sizes $(L=2000)$ and longer runs $\left(t_{s}=t_{r}=10^{6} \mathrm{MCS}\right)$ for obtaining the reference data at $\varepsilon=0$ in order to suppress the undesired effects of the diverging fluctuations and critical slowing down when approaching the above-mentioned critical point.

Our numerical analysis is repeated in the full payoff parameter region, which covers all social dilemma games at

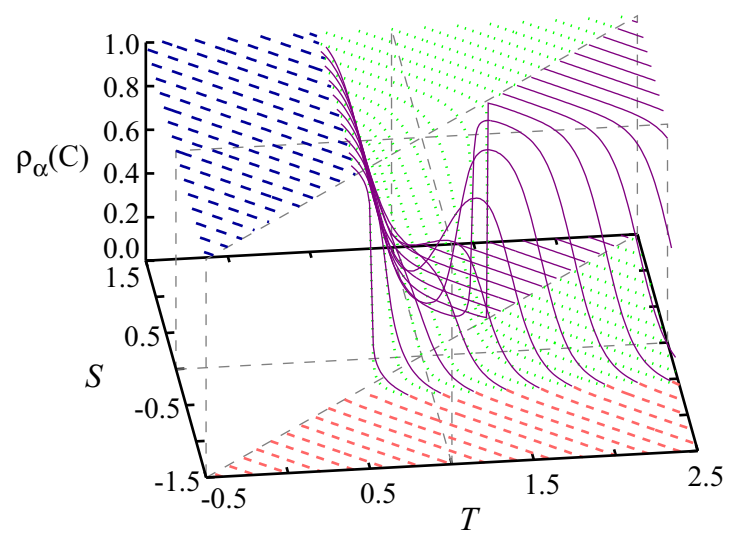

FIG. 5. (Color online) Frequency of strategy $C$ as a function of payoff parameters $T$ and $S$ in both sublattices for $K=0.3$ and $\varepsilon=0.1$. Blue and red lines (dark and light grey, thick dashed lines in printed version) illustrate MC data where the sublattices are equivalent. Purple (dark grey solid) and green (light grey dotted) lines show $\rho_{C}$ in the sublattices $X$ and $Y$ respectively.

fixed values of $\varepsilon$ and noise level $(K=0.3)$. The MC data are summed in Fig. 5, where the lines are obtained by varying $T-S$ at fixed values of $T+S$. The MC results indicate the absence of sublattice ordering, that is, $\rho_{X}(C)=\rho_{Y}(C)$, within large regions of the prisoner's dilemma, harmony, and stag hunt games. The $C$ strategies dominate equally both sublattices in the zero noise limit if $T<1$ and $(T-1-S)<0$ and the homogeneous $D$ state is stable if $S<0$ and $(T-1-S)>0$ for the zero noise limit $(K \rightarrow 0)$.

Staying at the same limit, only one of the sublattice ordered structures appears in the hawk-dove game quadrant $(T>1$ and $S>0$ ). More precisely, $\rho_{X}(C) \rightarrow 1$ and $\rho_{Y}(C) \rightarrow 0$ if $\varepsilon>0$ and $(T-1-S)<0$ or when $\varepsilon<0$ and $(T-1-S)>$ 0 . Evidently, when reversing the sign of $\varepsilon$ and $(T-1-S)$ separately, the preference between the two sublattice ordered structures are exchanged.

The similarity between the impact of staggered magnetic field in the Ising model and the introduction of a matching-pennies component in the hawk-dove game can be demonstrated by monitoring the motion of an interface separating the two sublattice ordered arrangements. For both cases the visualization of the evolutionary process shows clearly the expansion of the preferred sublattice ordered structure via the motion of separating interface.

It is emphasized that the twofold degeneracy can be observed only along the line $T-1-S=h=0$ separating regions where the chessboard and antichessboard arrangements of the $D$ and $C$ strategies occur, as shown in Fig. 5. It is noteworthy that Ising type critical transitions can take place only along this line if the noise level is increased.

When decreasing the values of $\varepsilon$ and $K$, the widths of the intermediate transition regions shrink, too. Additionally, one can observe a disordered state at close proximity to the central point ( $T-1=S=0$ ) of the $T-S$ plane, where both the coupling constant $J$ and the external magnetic field $h$ vanish in the equivalent Ising model. Evidently, the absence of ordering will also characterize the system behavior if $\varepsilon$ and/or $K$ exceeds threshold values depending on $T$ and $S$. 


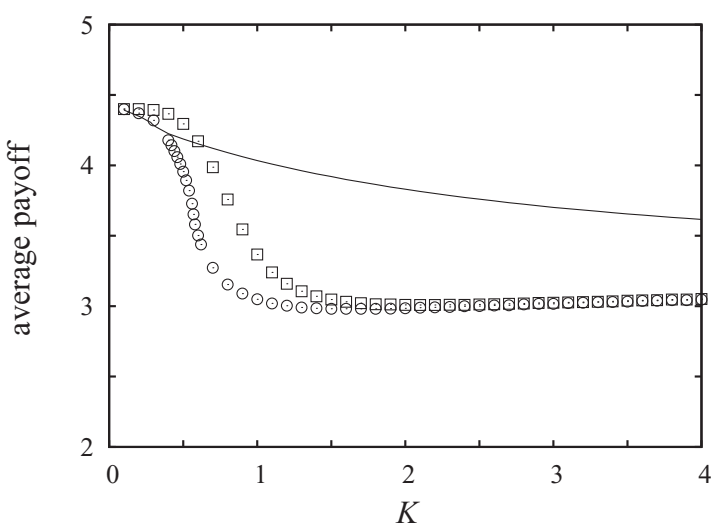

FIG. 6. Comparison of average payoffs versus noise $(K)$ for evolutionary games on square lattice at $T=1.9$ and $S=0.3$. Open circles and boxes represent MC data obtained for $\varepsilon=0$ and 0.1 if the evolution is controlled by the logit rule defined by Eq. (4). The solid line illustrates MC results obtained when the evolution is controlled by collective pairwise strategy update [54].

\section{EXPLOITATION OF CONGESTION}

The systematic analysis of the evolutionary games has highlighted the existence of many different mechanisms enhancing the total income of societies even for the cases of social dilemmas (for a survey, see [4,6,50,51]). It is already known that the total income of a society can be influenced by the game itself (payoffs and strategy set), the connectivity network, the dynamical rules including the noise level, and allowing coevolutionary processes in all ingredients of the systems. In the light of these results the utilization of congestion phenomenon emerges directly. The relevance of this question is stressed by recent experiments investigating human behavior in real-life situations involving the matchingpennies component in the payoffs $[31,52,53]$.

The above results justify that the main impact of matching pennies is related to the preference of one of the chessboardlike strategy arrangements that suppresses the critical transition related to the existence of two equivalent (optimal) ordered strategy arrangements. As a result, the preferred structure dominates the system behavior if $K>K_{c}$ within the region of hawk-dove games. The latter effect can increase the total income because of the reduction of the length of interfaces responsible to the loss in the total payoff. Conceptually similar congestion induced increase of collective income may be expected in human societies.

For an illustration of the mentioned phenomenon we compare the average payoffs as a function of noise $K$ for three different evolutionary rules, whereas the payoff parameters ( $T$ and $S$ ) are fixed. For the selected payoffs $(T=1.9$ and $S=0.3)$ the maximum average payoff $[2(T+S)]$ is achieved if the $C$ and $D$ strategies form a chessboardlike structure in the zero noise limit. It is emphasized that the matching-pennies component does not affect the average payoff because it is a zero-sum game.

Figure 6 illustrates that the presence of a matching-pennies component increases the average payoff for the given payoff parameters. The most relevant increase occurs in the vicinity of the critical noise level $\left[K_{c}(T=1.9, S=0.3)=0.590(4)\right]$. The importance of the congestion phenomenon becomes more striking when the average payoff is compared to results obtained for other types of dynamical rules. Within the hawkdove region the imitation-based rules cannot be considered as an adequate reference because the imitation of the neighboring strategy prevents the formation of the optimal strategy arrangement [55]. Up to now one of the highest average payoffs is achieved by the application of the collective pairwise strategy update [54], where the stochasticity is introduced via a noise parameter $K$, too. For the latter rule the fraternal players take into consideration the coplayer's income. The corresponding MC data of the average payoffs are illustrated by the solid line in Fig. 6. Accordingly, there is a region of $K$ where the introduction of the matching-pennies payoffs provide the highest average income for the whole population.

\section{SUMMARY}

In this paper we have studied two-strategy evolutionary games on a square lattice when matching-pennies games with a strength $\varepsilon$ are added to the payoffs in order to modify the interactions between neighboring players. Here a matchingpennies game represents the simplest cyclic dominant game for the description of the Red Queen effect in two-strategy games if the evolutionary process is controlled by the logit rule resembling the Glauber dynamics in statistical physics. In that case the matching-pennies component of the pair interactions can be considered as a microscopic driving force that destroys the detailed balance by inducing probability current loops throughout the four microscopic states for each pair interaction.

The presence of the matching-pennies component eliminates the equivalence between the players residing in the two sublattices. Additionally, the equivalence between the two ordered (chessboard- and antichessboardlike) strategy distributions is also destroyed via a contagion mechanism affecting the configuration probabilities if the disfavored strategy pairs [e.g., $(D, D)$ and $(C, C)]$ would be present with different probabilities at $\varepsilon=0$. The direction of preference varies with the sign of $\varepsilon$. Note that the presence of both ordered phases with equal weights is disadvantageous because along the separating interfaces frustrating players cannot enjoy sufficiently high payoff. This ambiguity can be resolved by the matching-pennies component in a desired way.

Finally we emphasize that similar cyclic dominance can be created by the rock-paper-scissors component in threestrategy evolutionary games for equivalent players, as reported for the description of many systems (for a survey, see Refs. [3,4,6,11]). In the latter cases, however, the matchingpennies components and their effects can be recognized within all the $2 \times 2$ subgames where the interacting players are limited to using two different strategy pairs. The latter feature underlines the importance of the matching-pennies games because it can be included within the $2 \times 2$ subgames for most of the symmetric $n \times n(n>2)$ matrix games. 


\section{ACKNOWLEDGMENTS}

This work was supported by the John Templeton Foundation (FQEB Grant No. RFP-12-22), the Hungarian National
Research Fund (Grant No. OTKA TK-101490), and the European Social Fund through project FutureICT.hu (Grant No. TAMOP-4.2.2.C-11/1/KONV-2012-0013).
[1] J. Maynard Smith, Evolution and the Theory of Games (Cambridge University Press, Cambridge, UK, 1982).

[2] J. W. Weibull, Evolutionary Game Theory (MIT Press, Cambridge, MA, 1995).

[3] M. A. Nowak, Evolutionary Dynamics (Harvard University Press, Cambridge, MA, 2006).

[4] K. Sigmund, The Calculus of Selfishness (Princeton University Press, Princeton, MA, 2010).

[5] J. von Neumann and O. Morgenstern, Theory of Games and Economic Behaviour (Princeton University Press, Princeton, NJ, 1944).

[6] G. Szabó and G. Fáth, Phys. Rep. 446, 97 (2007).

[7] M. W. Macy and A. Flache, Proc. Natl. Acad. Sci. USA 99, 7229 (2002).

[8] F. C. Santos, J. M. Pacheco, and T. Lenaerts, Proc. Natl. Acad. Sci. USA 103, 3490 (2006).

[9] D. Monderer and L. S. Shapley, Games Econ. Behav. 14, 124 (1996).

[10] D. Monderer and L. S. Shapley, J. Econ. Theory 68, 258 (1996).

[11] G. Szabó, K. S. Bodó, B. Allen, and M. A. Nowak, Phys. Rev. E 90, 042811 (2014).

[12] G. W. Brown, in Activity Analysis of Production and Allocation, edited by T. C. Koopmans (Wiley, New York, 1951), pp. 373-376.

[13] M. Voorneveld, Econ. Lett. 66, 289 (2000).

[14] L. E. Blume, Games Econ. Behav. 5, 387 (1993).

[15] L. E. Blume, Games Econ. Behav. 44, 251 (2003).

[16] J. Berkson, J. Am. Stat. Assoc. 39, 357 (1944).

[17] R. J. Glauber, J. Math. Phys. 4, 294 (1963).

[18] N. Metropolis, A. W. Rosenbluth, M. N. Rosenbluth, A. H. Teller, and E. Teller, J. Chem. Phys. 21, 1087 (1953).

[19] A. V. M. Herz, J. Theor. Biol. 169, 65 (1994).

[20] S. Galam and B. Walliser, Phys. A (Amsterdam, Neth.) 389, 481 (2010).

[21] G. Szabó, L. Varga, and I. Borsos, Phys. Rev. E 89, 042820 (2014).

[22] P. Morris, Introduction to Game Theory (Springer, New York, 1994).

[23] R. Cressman, Evolutionary Dynamics and Extensive Form Games (MIT Press, Cambridge, MA, 2003).

[24] J. Schnakenberg, Rev. Mod. Phys. 48, 571 (1976).

[25] L. Van Valen, Evol. Theory 1, 1 (1973).

[26] L. M. Van Valen, Evol. Theory 4, 289 (1980).

[27] D. Friedman, Econometrica 59, 637 (1991).
[28] R. Cressman, W. G. Morrison, and J.-F. Wen, Can. J. Econ. 31, 1101 (1998).

[29] J. Cremer, T. Reichenbach, and E. Frey, Eur. Phys. J. B 63, 373 (2008).

[30] A. Traulsen, J. C. Claussen, and C. Hauert, Phys. Rev. Lett. 95, 238701 (2005).

[31] Z. Cao and X. Yang, Theor. Comput. Sci. 540-541, 169 (2014).

[32] B. Xu, S. Wang, and Z. Wang, Eur. Phys. J. B 87, 46 (2014).

[33] M. Sysi-Aho, J. Saramäki, J. Kertész, and K. Kaski, Eur. Phys. J. B 44, 129 (2005).

[34] C. P. Roca, J. A. Cuesta, and A. Sánchez, Eur. Phys. J. B 71, 587 (2009).

[35] C. Domb, in Phase Transitions and Critical Phenomena, edited by C. Domb and M. S. Green (Academic Press, London, 1974), Vol. 3, pp. 357-484.

[36] R. B. Griffiths, Phys. Rev. Lett. 24, 1479 (1970).

[37] D. C. Rapaport and C. Domb, J. Phys. C 4, 2684 (1971).

[38] S. J. Penney, V. K. Cumyn, and D. D. Betts, Phys. A (Amsterdam, Neth.) 330, 507 (2003).

[39] C. Hauert and G. Szabó, Am. J. Phys. 73, 405 (2005).

[40] G. Kirchhoff, Ann. Phys. Chem. 72, 497 (1844).

[41] S. Katz, J. L. Lebowitz, and H. Spohn, J. Stat. Phys. 34, 497 (1984).

[42] B. Schmittmann and R. K. P. Zia, in Phase Transitions and Critical Phenomena, edited by C. Domb and J. L. Lebowitz (Academic Press, London, 1995), Vol. 17.

[43] G. Szabó, A. Szolnoki, and L. Bodócs, Phys. Rev. A 44, 6375 (1991).

[44] G. Szabó, A. Szolnoki, and T. Antal, Phys. Rev. E 49, 299 (1994).

[45] S. Katz, J. L. Lebowitz, and H. Spohn, Phys. Rev. B 28, 1655 (1983).

[46] R. Dickman, Phys. Rev. A 41, 2192 (1990).

[47] G. Szabó, A. Szolnoki, T. Antal, and I. Borsos, Phys. Rev. E 55, 5275 (1997).

[48] N. Sarkar and A. Basu, Phys. Rev. E 90, 022109 (2014).

[49] T. Chou, Biophys. J. 85, 755 (2003).

[50] M. A. Nowak, Science 314, 1560 (2006).

[51] M. Perc and A. Szolnoki, BioSystems 99, 109 (2010).

[52] M. Belot, V. P. Crawford, and C. Heyes, Proc. Natl. Acad. Sci. USA 110, 2763 (2013).

[53] Z. Cao, H. Gao, X. Qu, M. Yang, and X. Yang, PLoS One 58, e49441 (2013).

[54] G. Szabó, A. Szolnoki, M. Varga, and L. Hanusovszky, Phys. Rev. E 82, 026110 (2010).

[55] C. Hauert and M. Doebeli, Nature (London) 428, 643 (2004). 